\title{
Stochastic modelling of tyrosine kinase inhibitor rotation therapy in chronic myeloid leukaemia
}

\author{
H. Jonathan G. Lindström ${ }^{1}$, Astrid S. de Wijn² and Ran Friedman ${ }^{1 *}$ (I)
}

\begin{abstract}
Background: Resistance towards targeted cancer treatments caused by single nucleotide variations is a major issue in many malignancies. Currently, there are a number of available drugs for chronic myeloid leukaemia (CML), which are overcome by different sets of mutations. The main aim of this study was to explore if it can be possible to exploit this and create a treatment protocol that outperforms each drug on its own.

Methods: We present a computer program to test different treatment protocols against CML, based on available resistance mutation growth data. The evolution of a relatively stable pool of cancer stem cells is modelled as a stochastic process, with the growth of cells expressing a tumourigenic protein (here, Abl1) and any emerging mutants determined principally by the drugs used in the therapy.

Results: There can be some benefit to Bosutinib-Ponatinib rotation therapy even if the mutation status is unknown, whereas Imatinib-Nilotinib rotation is unlikely to improve the outcomes. Furthermore, an interplay between growth inhibition and selection effects generates a non-linear relationship between drug doses and the risk of developing resistance.

Conclusions: Drug rotation therapy might be able to delay the onset of resistance in CML patients without costly ongoing observation of mutation status. Moreover, the simulations give credence to the suggestion that lower drug concentrations may achieve better results following major molecular response in CML.
\end{abstract}

Keywords: Chronic myeloid leukaemia, Drug rotation, Treatment Simulation, Targeted therapy, Stochastic modelling

\section{Background}

Targeted therapies, which directly target molecular pathways critical to tumours instead of rapidly dividing cells in general, have in many cases improved survival significantly when compared to cytotoxic chemotherapy or radiation. Unfortunately, a recurring difficulty with targeted therapies is the occurrence of resistance [1]. As these therapies target oncogenic molecular pathways with high specificity, smaller, relatively common changes such as mutations in the molecular drug target, activation of alternate pathways and overexpression of the drug target or of transporter proteins, can render them ineffective [2].

*Correspondence: ran.friedman@Inu.se

'Department of Chemistry and Biomedical Sciences, Linnæus University, 391 82 Kalmar, Sweden

Full list of author information is available at the end of the article
Even if such a change occurs in a single cancer-cell it confers a fitness advantage that can generate a cell lineage which reproductively outpaces the rest of the tumour. In competition with the other cells, this gives such a lineage a higher probability of becoming a major fraction of the tumour cells [3]. This clonal evolution among the tumour cells allows major and minor resistance-giving traits to propagate in the population [4]. Once a large proportion of the tumour becomes resistant, the success of continued treatment is unlikely. In many cases, the fitness advantage which allows resistant clones to expand exists only during treatment. Thus, by altering the treatment protocol, it is possible that we could steer evolution towards a treatable state $[1,5]$. The vast number of possible protocols limit our ability to explore this in experiments. On the other hand, theoretical studies and computer modelling allow 
testing on an otherwise infeasible scale [6] and thus open a venue for estimation of multiple treatment protocols [7].

One of the first malignancies where targeted therapies proved effective was chronic myeloid leukaemia (CML) that involves the constitutively active tyrosine kinase Abl1. In most cases of CML, a chromosomal translocation creates $\mathrm{Bcr}-\mathrm{Abl}$, a fusion protein in which the Abelson tyrosine kinase $(\mathrm{Abl})$ is stripped of its regulatory regions, leaving the kinase domain free to catalyse any substrate it comes across, triggering numerous growth pathways as a result [8]. It has been shown that Bcr-Abl is sufficient for producing the malignant phenotype on its own. As the protein is both the sole driver and absent in healthy cells it is also an excellent drug target [9]. Imatinib, a BcrAbl specific tyrosine kinase inhibitor (TKI), was approved in 2001 and has become the standard CML treatment due to greatly improved outcomes over traditional therapy [10]. As it inhibits Bcr-Abl it effectively shuts down the signalling responsible for creating the malignant phenotype. In spite of this, Imatinib and related TKIs rarely cure CML. Instead, a minimal residual disease remains and most often patients continue treatment for the rest of their lives to prevent recurrence. This is thought to be caused by Bcr-Abl independent cancer stem cells, which carry the gene but do not require it [9].

A significant portion of Imatinib treatment failures are due to mutations which emerge in the kinase domain of Bcr-Abl that reduce the efficiency of Imatinib. To remedy this, a series of other TKIs have been developed that do not share the same resistance mutations and have an overall higher affinity for Bcr-Abl. These are most often used as a second-line treatment. For instance, the single nucleotide variations (SNVs) E255V and Y253H confer resistance towards Imatinib but not Dasatinib or
Bosutinib, respectively [11]. Until recently T315I was the only untreatable Abl1-SNV, because the mutated protein was resistant towards all available drugs. The Bcr-Abl inhibitor Ponatinib approved in 2012 has since changed that but is associated with more severe side effects than other TKIs, most notably vascular occlusion events, heart failure, and hepatotoxicity [12]. The EPIC trial comparing Ponatinib to Imatinib was terminated early due to arterial thrombotic events in some patients [13]. Thus, despite being less vulnerable to resistance mutations, Ponatinib is only given when no other options are available. Moreover, two mutations in the same copy of Abl1 (compound mutations) can cause resistance towards Ponatinib [14], and some SNVs, such as G250E and E255V, yield some degree of Ponatinib resistance (at least in vitro) [11].

The sensitivity of known mutants towards approved and experimental drugs was the subject of several studies (e.g., $[11,15,16])$. The results are often presented as $\mathrm{IC}_{50}$-values, i.e., the TKI concentration at which cell proliferation is slowed by $50 \%$. Mutations can change the drug binding protein such that the affinity for the drug is reduced, resulting in less effective inhibition. These data are a proxy for the fitness of individual mutants under treatment, and are used as such in this work.

The type of non-trivial protocols investigated in this paper are primarily drug rotations, where drugs are switched according to a fixed pattern as illustrated in Fig. 1. A range of both doses and timings were investigated. The drug rotations investigated maintained a constant drug pressure on the tumour, much like conventional treatments; but the source of this pressure changes over time. This is in contrast to the conventional treatment model, where a patient receives the same drug as long as the drug is tolerated and effective, and is moved to a

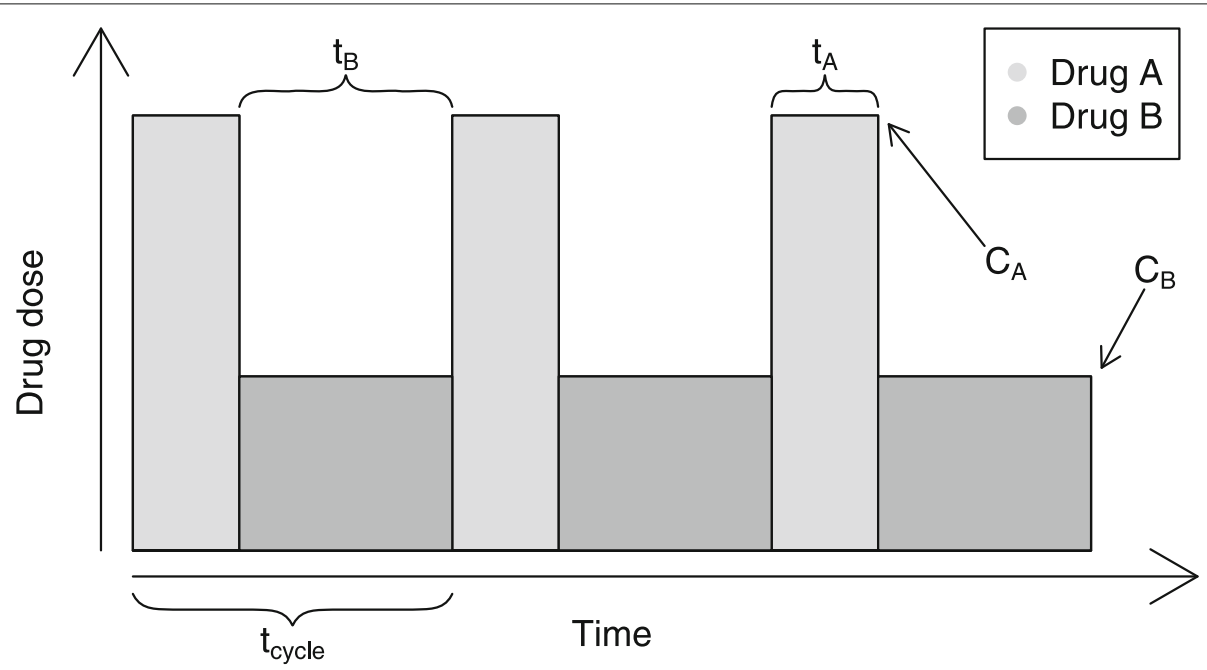

Fig. 1 Example of a drug rotation protocol. The protocol is defined by four variables: The drug doses $\left(C_{A}, C_{B}\right)$, and the time per cycle $\left(t_{A}+t_{B}=t_{c y c l e}\right)$ of either drug 
new drug (if available) otherwise. With Imatinib the dose can sometimes be reduced after an initial effect has been established [17]. It is however uncommon to terminate Imatinib treatment. Termination of treatment when active stem cells are still present is liable to cause a quick return of symptoms and increases the risk of disease progression, possibly via accumulating secondary adaptations, into accelerated phase or blast crisis with significantly worse treatment outcomes. Even with newer TKIs, there are as of yet no clear guidelines on the possibility of seceding treatment in CML [18].

On top of the potential benefits with respect to drug resistance, a drug rotation can help manage side effects, which is especially pertinent for Ponatinib, while also lowering the overall risk of resistance. If the two drugs have little or no overlapping resistance mechanisms the likelihood of adapting to both may be lower, while singly resistant cells are still exposed to one drug towards which they are sensitive. However, there is also a risk for development of mutations that make the molecular drug target resistant to both drugs. In principle, combination therapies where several molecular targets are treated at the same time are beneficial in this aspect [19], but such therapies are not currently available for CML. Using multiple Bcr-Abl TKIs simultaneously means they have to compete with one another, as they all target the same binding pocket of the enzyme. Thus such combination therapy is expected to be less efficient in eradicating the cancer cells. Because of these factors it is unlikely that combinations would allow for significant dose reductions, and because of side-effect concerns in giving multiple TKIs simultaneously we decided not to pursue it in this work.

In this paper, we investigate whether drug rotation protocols using TKIs for the treatment of CML could reduce the risk of resistance. It is shown that drug rotations with Ponatinib have the largest potential benefits. Furthermore, the risk of developing resistance seems tied to the achieved degree of inhibition with reduced risks for both low $(<40 \%)$ and high (>90\%) degrees of inhibition.

\section{Methods}

\section{Modelling approach and assumptions}

If the growth rate of any particular mutant subject to treatment is known, it becomes possible to simulate the evolution of CML cells under a time-varying treatment protocol. Since cell growth and mutation are not entirely deterministic, the evolution of CML cells is appropriately modelled as a stochastic process. The derivation of such a model enables the investigation of potentially superior non-trivial treatment protocols for CML.

The branching process [20] models cell growth as a stochastic process where individual cells at the end of their life give birth to (possibly mutated) offspring according to some probability distribution. Branching processes have previously been used to (among many examples): optimise screening in ovarian cancer [21], evaluate the effects of combination therapy [19] and numerous other modelling instances of treatment response and resistance prevention [22]. We propose a model similar to the standard branching process, albeit slightly restructured for more convenient computer simulation. Furthermore, we consider only a non-hierarchical, stable number of cancer cells (but with fluctuations about some mean) capable of self replication (cancer stem cells, CSCs). CSCs are important in modelling of cancers [23] and in particular in CML [24]. It should be noted that in cells that do not self-replicate (i.e., they are not CSCs) there can be no lasting adaptations, as their lineage is bound to die and no traits can be permanently acquired. If the CSCs replicate in a strict hierarchy the population capable of sustaining a mutation is further reduced [25]. The CSC pool also grows more slowly than differentiated cells [24] and the cells are considerably less sensitive to Abl1 inhibitors [26], motivating our choice to model the CSC pool as stable even under treatment. To that end, we introduce an adaptive death rate which means that the model proposed here is not equivalent to a typical branching process. In the context of this model, we judge resistance progression by the makeup of the CSC pool. A common assumption is that CML CSCs are entirely insensitive to TKIs [24, 27]; However, experimental studies show that while TKIs cannot kill CSCs, they have some degree of antiproliferative effect $[28,29]$, a phenomenon that our model replicates.

\section{Computational model}

The discrete time model of stochastic cell growth proceeds essentially as follows. Initially, a population of cells is set up. A simple two-step procedure is then repeated iteratively: (each step will be described in further detail below)

1. Each cell has a chance to die.

2. Each of the surviving cells has a chance to reproduce, with a small probability of producing a mutant.

Treatment protocols are simulated by letting the reproduction probability change with time in a way that depends on the mutation status of the cell. A pseudocode description of the algorithm is available in Section 8 of Additional file 1.

In every iteration, there is some probability $q$ for each cell to die. The cells that survive reproduce with a probability $s_{i}$ that depends on their genotype $i$. To reduce the computational burden, all cells of an identical genotype are treated together, and thus the total number of deaths or births for one group per iteration is described by the binomial distribution $B(n, p)$ for $n$ independent but identical cell events (i.e. births/deaths/mutations), and 
probability $p$ (which can be birth-/death- or mutationprobability). Under the effect of a single drug, the birthprobabilities, i.e. the odds of a cell of type $i$ dividing in a particular timestep (analogous to a birth rate in a continuous setting) are calculated as

$$
s_{i}(t)=s_{i}^{(0)} 2^{-C(t) / I C_{50}}
$$

where $C(t)$ is the time-dependent drug concentration (as exemplified in Fig. 1), and the $\mathrm{IC}_{50}$ depends on both genotype and drug. $\mathrm{IC}_{50}$ values are taken from [11], where these values are available for all single mutations and some, but not all compound mutations. This use of $\mathrm{IC}_{50}$ values is discussed further in Additional file 1: Section 4. In cases where $\mathrm{IC}_{50}$ values for compound mutations are not available, they are estimated as the maximum $\mathrm{IC}_{50}$ of the mutation's constituent SNVs which is a decent approximation in many cases (Additional file 1: Figure S2) when no other data exists, though it is known that some compound mutations are much more highly resistant than expected [14]. The starting birth-probabilities $s_{i}^{(0)}$ can be unified or set separately for each genotype. A method for deriving these from position specific scoring matrix (PSSM) data is provided in the Additional file 1: Section 1. However, the rate of evolution in the presence of drug therapy is in fact almost independent of the background reproduction rate of drug-free tumour cells, since the presence of drug therapy alters the evolutionary landscape to a much greater degree. Differences in the inherent growth rate are thus insignificant compared to the much larger reproduction rate differences induced by treatment. On the contrary, if periods of treatment-free growth were to be simulated, variations in reproduction rate would likely be important [30].

Under the assumptions that only cancer stem cells lead to resistant clones [24] and that these cells are rather resistant to the drugs [9] the effective population size is kept stable, though fluctuations about the mean are allowed. To keep the population size average fixed, the deathprobability is rebalanced at the start of every iteration

$$
q(t)=\frac{\sum_{i} N_{i} s_{i}(t)}{N}\left(1+\kappa \frac{N-\hat{N}}{\hat{N}}\right) \frac{N}{N+\sum_{i} N_{i} s_{i}(t)}
$$

$N$ is the total population size, $\hat{N}$ is the desired mean and $\kappa$ is a spring constant describing how strongly $N$ tends towards $\hat{N}$ (Table 1$). \sum_{i} N_{i} s_{i}(t)$ is the expected number of new cells (since $E[B(n, p)]=n p$ ). There are three components to this equation. The first factor is simply the average birth rate at that timestep which, in a deterministic setting, is what the death rate should equal for the population to remain constant. The second factor is used
Table 1 Global parameters

\begin{tabular}{lll}
\hline Parameter & Symbol & Value \\
\hline Population size avg. & $\hat{N}$ & 250000 cells [24] \\
Population size spring constant & $\kappa$ & 1.0 \\
Mutation rate multiplier & $\mu$ & $10^{-7}$ mutations/division $^{\mathrm{a}}$ \\
Unmodified growth rate & $s^{(0)}$ & 0.016 divisions/timestep ${ }^{\mathrm{b}}$ [30]
\end{tabular}

aThe number of mutations in a single residue per new cell where only one transversion can result in the specific amino-acid change. Mutations that result from transitions or more than one possible SNV have higher mutation rates, see Additional file 1: Section 5 and Table S1

${ }^{b}$ Corresponds to approximately $0.3-0.4$ divisions per day

to increase or decrease the death rate when the population size is too high or low respectively. This causes the population size to drift towards $\hat{N}$ as shown in Figure S1 in Additional file 1; how quickly it does so depends linearly on the difference between $N$ and $\hat{N}$. Finally, the last factor accounts for the drift otherwise induced by the algorithm (killing cells first and then letting them reproduce). The population size oscillates up and down as cells reproduce and are killed in turn; this factor makes sure that the population size is $\hat{N}$ on average at the start of each timestep.

Whenever a new cell is produced it has a small chance $\mu_{i}$ of being a mutant. Only single nucleotide variations (SNVs) are allowed in a single cell division (though they may accumulate and form compound mutations over time). Mutation probabilities are specified at a genome level and includes altered transition/transversion ratios $(\mathrm{ts} / \mathrm{tv}=2)$. Explicitly modelling the genetic code for possible resistance mutation spots avoids some potential cases of a residue-residue transition probability matrix. This, and the multiplier $\epsilon_{j i}$ are explained in further detail in Section 5 in Additional file 1. Mutations that result in a variant with a known drug sensitivity are kept. Synonymous mutations are also kept. All other mutations are assumed to be deleterious or irrelevant and are not accepted. Backwards mutations that restore the wildtype from a mutant are allowed.

Taking only the leading order behaviour of growthand death-rates into account, the average the change in population for a genotype in one iteration is

$$
\begin{aligned}
N_{i}(t+1)= & \overbrace{\left[1+s_{i}\left(1-\mu_{i}\right)\right][1-q(t)] N_{i}(t)}^{\text {Growth term }} \\
& +\underbrace{\sum_{j \in n n_{i}} s_{j} \mu \epsilon_{j i} N_{j}(t)[1-q(t)]}_{\text {Mutation term }}
\end{aligned}
$$

where the sum in the mutation term is over all other genotypes that could mutate into $i$ (nearest neighbours, $n n_{i}$ ). Let $\mathbb{P}[\ldots]$ denote the probability of an event. Since 


$$
\epsilon_{j i} \equiv \frac{\mathbb{P}[j \text { mutates to } i]}{\mathbb{P}[\text { Single transversion }]}=\frac{\mathbb{P}[j \text { mutates to } i]}{\mu}
$$

and $\mu$ is a constant mutation rate that depends on the behaviour of the cancer cells (see Table 1), $\mu \epsilon_{j i}$ is the chance of $j$ mutating to $i$ specifically and

$$
\mu_{i}=\sum_{j \in n n_{i}} \mu \epsilon_{i j}
$$

is the chance of any specific residue mutating. A single transversion is chosen as the baseline since is the most unlikely type of SNV. Higher order terms appear in Eq. (3) if population size fluctuations are taken into account. When the drug concentrations are stable, the waiting time between events will effectively have a geometric distribution, which is the discrete analogue of the exponential waiting times characteristic of Poisson processes. Thus, the cells have no memory of whether they were recently changed by a mutation or not.

\section{Implementation}

All simulations were run using the same global parameters (Table 1); $\hat{N}$ and $\mu$ were selected based on conditions that are plausible for a newly diagnosed patient. We assume that CML is driven by a relatively large $\left(2.5 \cdot 10^{-5}\right.$ cells $)$ CSC pool with no clearly defined internal hierarchy. If CML has a more distinct hierarchic structure, the relevant CSC pool might be vastly smaller as only the most basal (stem-cell like) cells can sustain a mutation [25, 27]. In the case of small population sizes, stochastic effects dominate the evolution [31], and effects from the treatment prototol, if at all present, will be harder to detect.

By correlating the time until Imatinib resistance would generally occur in practice $[32,33]$ and the time it takes for a rare mutation to grow to a major fraction of the CSC population [34] with simulations, these parameters were determined to result in timesteps that are about $1.2 \mathrm{~h}$ long (600 timesteps/month). Simulations were started with Bcr-Abl cells where $100 \%$ of the cells carried Abl1 with wildtype kinase domain. $\mathrm{IC}_{50}$ values for known resistance mutations are taken from [11]. It is not known for certain whether resistant cells exist prior to treatment $[35,36]$ but the results starting from a pure wildtype population are still valid subject to the condition that the resistant cells are a minor fraction of the population when treatment starts (which is essentially equivalent to removing the waiting time for mutations to occur). The effects from drug-rotations on controlling the outgrowth of extant mutations remains the same.

There are several ways to simulate drug doses explicitly. One approach is to refer to the concentration of the drugs in the plasma which is available from experimental measurements. This approach, however, is not without limitations, as the treatment effects of available drugs do not match the achieved blood plasma concentration very well [37, 38]. One mechanism for this discrepancy could be that the drugs bind to a different degree to serum proteins, as observed with other small molecule drugs [39]. For instance, TKI doses based on blood plasma concentrations incorrectly indicate that Nilotinib is superior to any other available CML drug, as its achievable plasma concentration is over 100 times its wildtype $\mathrm{IC}_{50}$, and high enough that it should be effective against known resistance mutations. In practice, however, there are resistance mutations that make Nilotinib inactive. Owing to these limitations, we approximate the drug doses by the percent inhibition they achieve in vivo. This cannot be directly correlated to available experimental results, but could in principle be measured in cell-lines that are harvested directly from patients.

Drug doses were modelled as constant throughout the simulations. It might be argued that a wave-form is more suitable to represent drug doses. While this is true in some cases (e.g., drugs that are given once per week or less often and are slowly absorbed, or drugs that are rapidly cleared from the body), a constant value is used here owing to the frequent medication (at least once per day) and the relatively slow clearance of most the drugs. Thus, they establish a reasonably stable dose over a few weeks and the cycle times considered here of (1-4 months) are well above that. The exception is Dasatinib with a short half life of only $4 \mathrm{~h}$ [40] but even dasatinib is not completely eliminated from the body within a week after a single dose (data taken from the registry of Pharmaceutical Specialists in Sweden [41]).

Some software implementation details are provided in Additional file 1: Section 2.

\section{Results}

As a general measure of when resistance occurs we define $\mathrm{WT}_{\frac{1}{2}}$ as the number of iterations before the wildtype makes up half of the total population.

$$
\mathrm{WT}_{\frac{1}{2}} \equiv \text { timesteps before } N_{w t}<\frac{N}{2}
$$

Stochastic effects are most significant for low population phenotypes, and at $\mathrm{WT}_{\frac{1}{2}}$ it is very likely that the total population consists mostly of a few highly populated phenotypes. These grow rather deterministically, which means that estimated $\mathrm{WT}_{\frac{1}{2}}$ values do not display large stochastic fluctuations.

\section{TKI dose scaling effects}

Figure 2 displays the rate of evolution of tumour cells as a function of percent inhibition, under different models: pure fitness effects (Moran model, Fig. 2a), our numerical model as described below (Fig. 2b), and a theoretical model, where any resistance mutation is guaranteed to be fixed (Fig. 2c, model details in Additional file 1: Section 3). 


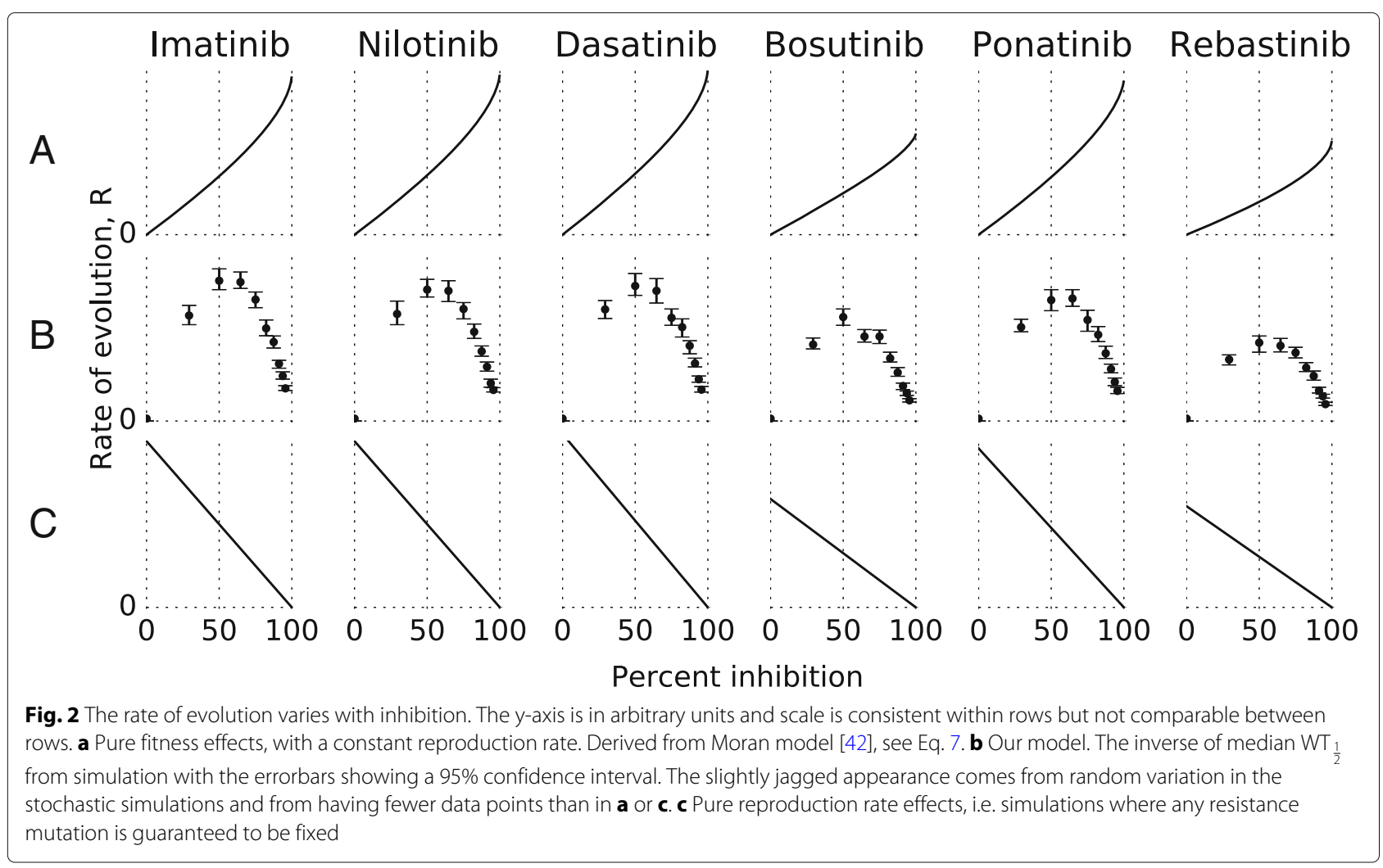

In our model, simulations were run at doses that correspond to an (initial) growth rate inhibition of $29 \%-96 \%$ for all drugs where the required $\mathrm{IC}_{50}$ data could be obtained (Fig. 2b). The results were then used to examine the effects of TKI doses on the overall rate of evolution $R$ i.e., the rate at which mutants become dominant in the population. It is assumed that under these conditions the most dominant population of cells will eventually become evolutionary fixed if the tumour grows for enough generations. This may or may not happen within the patient's lifetime. For practical reasons fixation in simulations is therefore estimated using the $\mathrm{WT}_{\frac{1}{2}}$ concept (Eq. 6). Thus, we refer to populations that have reached $\mathrm{WT}_{\frac{1}{2}}$ as being fixed, and to the probability to reach $\mathrm{WT}_{\frac{1}{2}}$ as the fixation probability. Explicitly, $R$ is calculated as:

$$
\begin{aligned}
R= & \text { (near) fixation probability } \times \text { mutation rate } \\
& \times \text { reproduction rate }
\end{aligned}
$$

Bosutinib and Rebastinib have a somewhat lower rate of evolution, due to the smaller number of known, however weak, resistance mutations. In the case of Rebastinib, this is likely because its different binding mode means the usual resistance mutations are less relevant, and as it is not clinically approved there is almost no clinical data on resistance. Of note, a similar bias may exist for other drugs (except Imatinib), as the in-vitro screening of mutants is based around mutations known primarily from
Imatinib-treated patients. Ponatinib is in practice only vulnerable to compound mutations. In vitro studies reveal that many mutations confer some resistance to Ponatinib [14], but the percent inhibition that is achieved in patients is apparently enough to make the inhibitor useful anyhow. In effect, this means that a standard Ponatinib treatment results in very high percent inhibition and thus low rates of evolution. Interestingly, this does not affect the shape of the curve(s) in Fig. 2. Note that the maximum rate of evolution occurs slightly above $50 \%$ inhibition in our model (Fig. 2 row b) regardless of the inhibitor. While absolute rates are highly dependent on the list of mutations provided, the percent inhibition that leads to maximum of $R$ appears to be independent of the actual mutations.

Two interacting effects can be seen in Fig. 2.

1. As drug doses increase, the fitness advantage of resistant mutants grows (Fig. 2a). This increases the rate of evolution as resistance mutations are more likely to be fixed (of note, these results are derived with the Moran model [42], with the additional assumption that deleterious mutations cannot become fixed). Because of the large population size (Table 1) and since in the small set of mutations considered there are few deleterious mutations, their contribution is negligible. If we assume a constant reproduction and mutation rate, the rate of evolution depends only on the probability of fixation for all the 
relevant SNVs. Mutations are rare enough that usually no more than one (relevant) SNV is present in the population at one time. Thus the rate of evolution for any mutation is approximately proportional to the sum of each individual SNVs fixation probability in a population of wildtype cells

$$
R \propto \sum_{i \in \mathrm{SNVs}} f_{i} H\left(f_{i}\right) \quad \text { where } f_{i}=1-\frac{2^{C / I C_{50}^{(i)}}}{2^{C / I C_{50}^{(w t)}}}
$$

which, given these assumptions, is directly proportional to the rate of evolution. $H(x)$ is the Heaviside step function:

$$
H(x)= \begin{cases}0 & x<0 \\ 1 & x \geq 0\end{cases}
$$

2. As drug doses increase, the overall reproduction rate slows down. Fewer cell divisions lead to fewer mutants which leads to a slower rate of evolution (Fig. 2c).

Unlike the reproduction rate, which can be slowed to an asymptotic halt, as drug doses go towards infinity fitness effects can never speed up evolution beyond a certain limit. With increasing selective advantage the probability of fixation of any resistance mutation, however weak, approaches 1 . Thus the rate of evolution becomes entirely limited by how often resistance mutations occur. This results in the peaks evident in row b of Fig. 2, where at a medium degree of inhibition (slightly above 50\%) reproduction rate is high enough to produce a significant number of mutants, and mutant fitness is high enough that the mutations are likely to be fixed once they occur. The rate of evolution then sharply decreases and a higher degree of inhibition results in a lower risk of resistance mutations, consistent with [43], who studied response to the degree of inhibition. At the lower inhibition end, this lends some extra credence to the idea of lowering doses for patients in major molecular response (MMR, a treatment response criterion based on very low levels of Bcr-Abl1 transcript) [17]. It seems that benefits might extend beyond lowering side effects into slowing down SNV-based resistance. It is however relatively well established [44] that higher doses create a more effective and lasting response. For initial treatment it is still likely that aiming for the highest achievable inhibition is the superior strategy even if it increases the risk of mutations. Apoptosis induced proliferation [3], where dying cells signal their neighbours to reproduce faster, can modify such models but is not considered here. Likewise, whether very low doses compare favourably to no treatment at all is not addressed by this model.

\section{TKI-rotation protocols}

TKI-rotation protocols with Imatinib-Nilotinib, and Bosutinib-Ponatinib were selected for examination. Our hypothesis was that drugs with more significant difference in their resistance mutation spectrum would be better candidates for rotation therapy. The treatment protocols were represented by interlaced square-waves of the two alternating drugs as shown in Fig. 1. These protocols were tested at a range of doses and timings as displayed in Figs. 3 and 4. Consistent with our hypothesis, there appears to be more potential benefits in BosutinibPonatinib rotation, in contrast to Imatinib-Nilotinib rotation which appears roughly equivalent to constant protocols of either drug. The benefits are not universal to any dose-timing combination, though it appears possible to achieve a therapeutic benefit under a wide range of conditions. Introducing low dose (29\%) Bosutinib always reduces the chance for resistance as compared with pure Ponatinib. However, such a concentration is probably too low to effectively treat active CML (prior to MMR). To a lesser degree, introducing a medium-high dose (75\%) of Ponatinib also appears to be superior to pure Bosutinib in terms of resistance, but perhaps not side effects and toxicity. At $50 \%$ inhibition a Bosutinib-Ponatinib rotation slows the emergence of resistance by at best $7.5 \%$ or $36 \%$ over pure Bosutinib or Ponatinib respectively (Fig. 5). Thus, compared to switching drug upon resistance, a rotation treatment protocol would remain effective for about 4 months longer than the best constituent monodrug protocol. To examine the effect of a reduced population size, the simulations where repeated with $\hat{N}=400$, as was used to model haematopoesis [31, 45]. No benefical effects were observed in that case (Section 9 and Figure S4 in Additional file 1). This could be because the increased randomness in when a mutation occurs obscures any effect. Another possibility is that cycle length was not optimal; in contrast to the large population where cycle length does not seem very important (see below) shorter fixation times in the small population might necessitate shorter cycles. However, as most estimates of the CSC populations size are almost three orders of magnitude larger than $\hat{N}=400$ we did not examine this further.

Our results are consistent with recent clinical studies. A rotation of Bosutinib and Ponatinib, has been applied successfully in a multi-resistant patient [46], whereas a rotating protocol of Imatinib and Nilotinib was equivalent to standard therapy in its treatment effect [47]. Note also that even rotating protocols which do not have any significant improvement in the treatment effect still have benefits for side-effect management. The Bosutinib-Ponatinib rotation seems beneficial in tumours that exhibit wt Bcr-Abl under the right circumstances according to our model. Inhibition for a multitude of other combinations with 


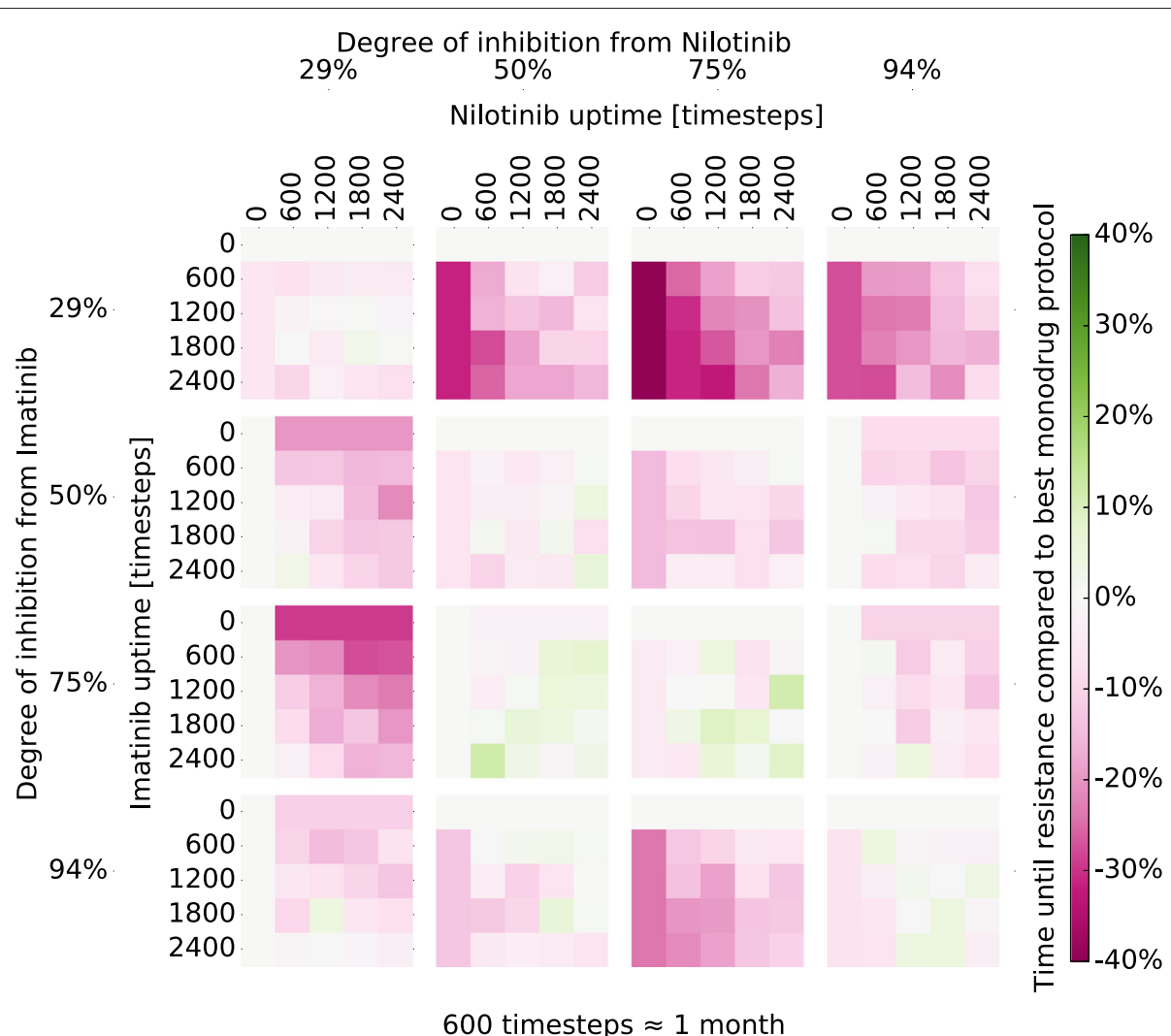

Fig. 3 Relative change in $\mathrm{WT}_{\frac{1}{2}}$ for Imatinib-Nilotinib rotation protocols. Each smaller heatmap shows the effects of different rotation timings at a certain dose of each drug. For instance, the (600 Imatinib, 1200 Nilotinib) box in the (50\%, 50\%) heatmap shows the effect of a drug rotation with $t_{a}=600$ timesteps of Imatinib treatment (ca. 1 month) followed by $t_{b}=1200$ timesteps of Nilotinib treatment (ca. 2 months), see Fig. 1, with both drug concentrations set such that cell growth was slowed by $50 \%$. In half the simulations the rotation started with Imatinib, and in the other half it started with Nilotinib. The colours correspond to the change in $\mathrm{WT}_{\frac{1}{2}}$ compared to a constant Imatinib or Nilotinib, whichever is better, with the same degree of inhibition. A zero duration uptime of one drug implies a constant concentration of the other. Zero-zero uptime boxes are set as $0 \%$, as they cannot be assessed since drug resistance almost never occurs without selective pressure from drugs

varying degrees of potential benefit was also tested and the results are shown in Additional file 1: Figures S3a-S3j.

In general, the degree of inhibition and the timing ratios seem to be the biggest factors in determining the effect of a Bosutinib-Ponatinib rotation. The former is evident from Fig. 4. The latter is shown in Fig. 5 which demonstrates that protocol effects at a fixed degree of inhibition is mainly a function of the timing ratio. If we define $x$ as the fraction of time dedicated to one of the drugs in a cycle (horizontal axis in Fig. 5), and we let

$$
\psi_{i}=1-\frac{2^{-(1-x) C_{A} / I C_{50}^{(w t)}-x C_{B} / I C_{50}^{(w t)}}}{2^{-(1-x) C_{A} / I C_{50}^{(i)}-x C_{B} / I C_{50}^{(i)}}}
$$

where $C_{A}$ and $C_{B}$ are concentrations of the respective drugs and the fitting parameters $k$ and $m$ are set such that the end-points match the $\mathrm{WT}_{\frac{1}{2}}$ of pure Ponatinib or Bosutinib treatment, and

$$
\phi_{i}=\psi_{i} H\left(\psi_{i}\right)
$$

where $H(x)$ is the Heaviside step function, then, the median $\mathrm{WT}_{\frac{1}{2}}$ (Eq. 6) is approximately described by

$$
\mathrm{WT}_{\frac{1}{2}}(x)=\frac{k}{\sum_{i \in n n_{w t}} \epsilon_{w t, i} \phi_{i}}+m
$$

Recall that $\epsilon_{j i}$ describes how likely a particular mutation is to occur (Eq. 4) and the summation is carried out for all residues that can be mutated (nearest neighbours, vide supra). Intuitively, $\mathrm{WT}_{\frac{1}{2}}$ depends on the fixation probability of each of the possible mutations and how often they appear, all the while assuming fixation time is so much slower than drug rotation that the effects of two drugs can be incorporated. The fitness of any particular mutation will change with $x$; if it is resistant towards one drug but not the other then at some value of $x$ it goes from being resistant to neutral and its fixation probability, $\phi_{i}$, goes from a finite positive number to zero. The 

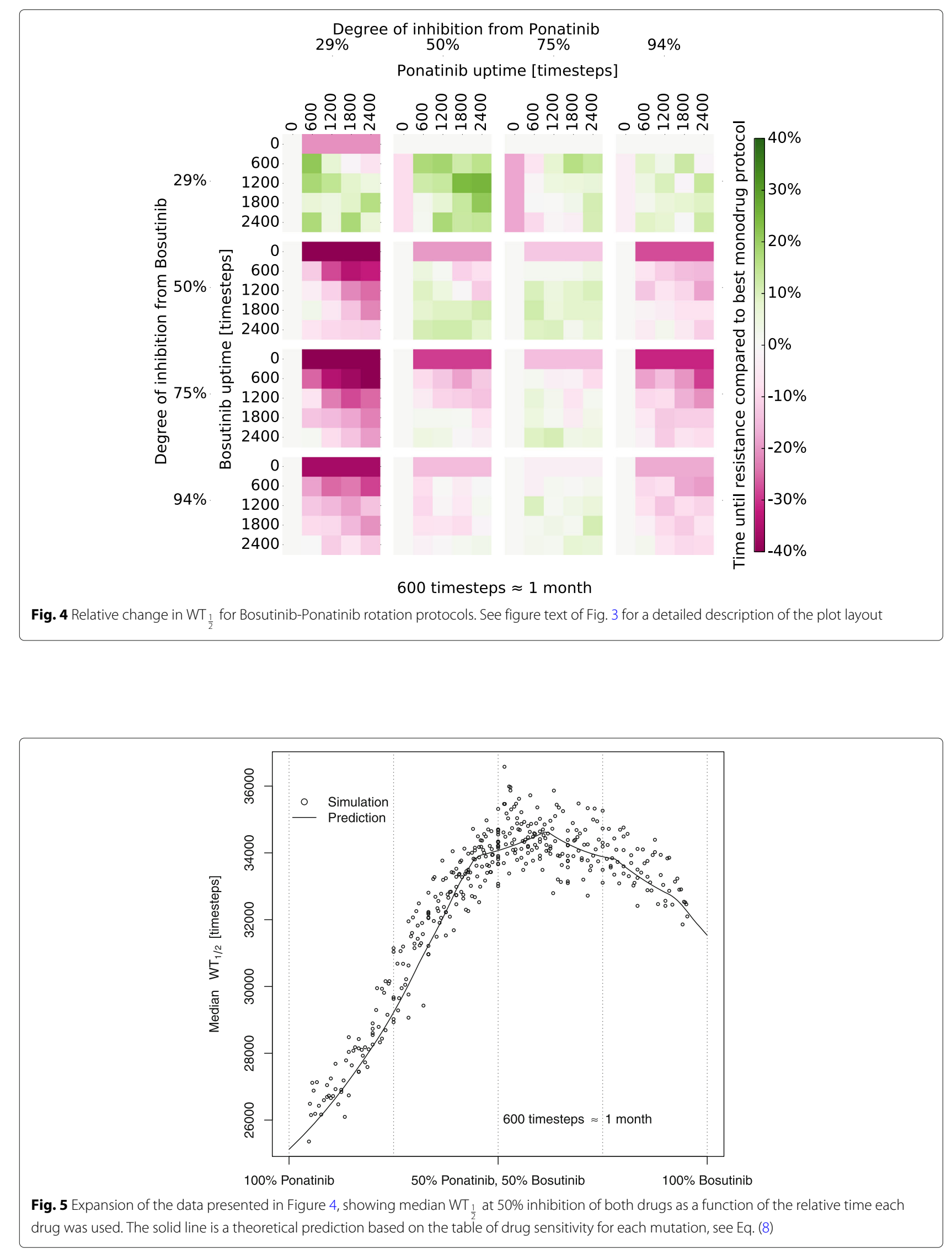
full derivation is provided in Additional file 1: Section 6. Combining these effects from all possible mutations, and taking into account how often they appear (proportional to $\left.\epsilon_{w t, i}\right)$, results in Eq. 8 and the predictions shown in Fig. 5 and Figures S3a-S3f in Additional file 1. The irregular bumps in the curve occur when any particular mutation goes from being more resistant than the wildtype to being less resistant or vice versa; the large number of known mutations means that this happens several times for most drug combinations. No correlation could be found for cycle length within the tested intervals but some correlation must exist, since very long cycles approximate a constant protocol. Equation (8) is not valid for those cases as it assumes fixation time is much longer than cycle time.

The benefits of a rotation protocol are due to the positive (though small) probability that non-polyresistant mutants are exposed to a drug towards which they are sensitive before they can grow to become a significant part of the population. At best this eliminates them outright, but should that fail their growth can be slowed and replacement significantly delayed. Evolutionary studies suggest that when a new mutation occurs right before a drug switch, it is likely to be subsequently eliminated when the fitness landscape changes [48]. Another important effect of a shifting fitness landscape is a reduced effectiveness of selection. This is both beneficial and detrimental for this type of protocol design, for while it reduces the advantage of resistant clones it also makes it more unlikely for them to be eliminated.

TKI-rotation protocols also seem to have an effect on the resulting distribution of observed mutations. Monodrug protocols of Bosutinib and Ponatinib and a drug rotation were simulated until a mutant had taken over in each simulation, and the most common mutation at $\mathrm{WT}_{\frac{1}{2}}$ was recorded. The resulting mutant distribution is shown in Fig. 6. As is evident the rotation protocol can, somewhat counter-intuitively, cause any type of change in distribution, and is not limited to an interpolation between the two constant protocols. It is perhaps possible to exploit this such that evolution moves towards mutants where effective inhibitors are available. Whereas the example in Fig. 6 favours G250E and E255K, both of which are at least somewhat resistant to all available drugs, does not fall into this category, it is possible that some combinations would steer evolution towards more easily treatable mutations. For instance, if a highly effective drug existed against G250E and E255K which are made more common by the rotation in Fig. 6, the combination would effectively steer evolution towards a treatable set of resistance mutations with an increased probability. Indeed, if the onset of resistance cannot be effectively prevented or delayed, ensuring

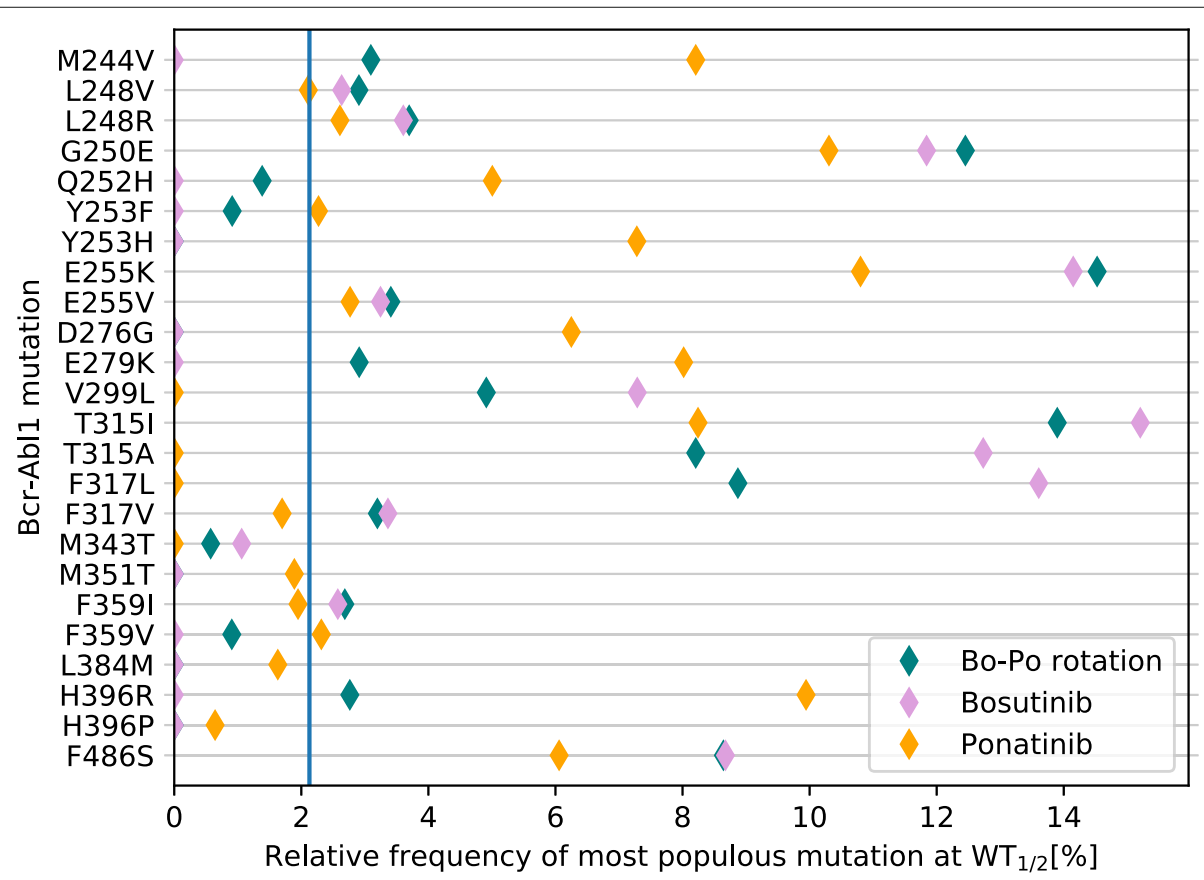

Fig. 6 Distribution of most common mutation at $\mathrm{WT}_{\frac{1}{2}}$ for three protocols, simulated 50000 times each. The protocol has a significant effect on mutant distribution $\left(\chi^{2}\right.$-test, $\left.\chi^{2}=56929, p<10^{-16}\right)$. The vertical blue line shows the expected frequency assuming all mutations were equally likely and equally fit. Unobserved mutations and double (or higher) mutants are not shown. For these simulations, doses were set such that Bosutinib caused 50\% inhibition and Ponatinib caused 75\% inhibition. The simulation protocol was 3:1 Bosutinib:Ponatinib, i.e., Bosutinib was used during 1800 timesteps (ca. 3 months), followed by Ponatinib during 600 timesteps (ca. 1 month) etc 
it happens in a less harmful way can still provide some benefit.

\section{Discussion}

We have developed, implemented and tested a model for simulating treatment protocols based on available $\mathrm{IC}_{50}$ data. Using $\mathrm{CML}$ as an example we have shown that drug rotation therapy with available Bcr-Abl TKIs could potentially decrease the risk of resistance. These are some suggested guidelines as to when it may be better that standard therapy:

- The drugs have different resistance profiles.

- There is a sub-optimal drug response from a molecular point of view, i.e. the amount of inhibition achieved places the patient in the zone where resistance happens the fastest (ca. 50\% inhibition, Fig. 2).

The first condition is fulfilled by different combinations of Abl1 inhibitors, e.g., Nilotinib + Dasatinib and Bosutinib + Ponatinib. As for the second condition, it is clear that all drugs reduce the number of tumour cells efficiently, but some persistent cells seem to survive (at least with Imatinib, which is the most common inhibitor). In addition, a rotation protocol may be useful if an inhibitor is working fine but leads to difficult side effects. Furthermore, the timing ratio appears to be more important than exact cycle length, so cycle length could be optimised for side effect reduction or other factors.

In developing the model, we chose to use a stable population size. This choice is based on the assumption that cancer stem cells, that drive the evolution within the tumour, are never eliminated by TKIs though their proliferation may be hindered. It should be noted, however that the results could be influenced by this assumption. Another necessary limitation of the model is that $\mathrm{IC}_{50}$ values are not always consistent. Measured $\mathrm{IC}_{50}$ values vary significantly between studies [49]. On the other hand, there is evidence that they have clinical relevance for drug selection [50]. Compound mutations are included in the model. $\mathrm{IC}_{50}$ values are available for those that are most clinically relevant, whereas in the other cases we made the assumption that the double mutant is as resistant as the most resistant single mutation. Yet another limitation is ignoring the effect of pharmacokinetics and treating drug doses as constant. Patients do not experience a truly constant drug dose in practice, and it is known to have an effect under other circumstances [51, 52]. Investigating the effects of this is a potential direction for future studies.

\section{Conclusions}

The potential delay in the onset of resistance has to be weighed against the risk of more severe side-effects. The greatest gains predicted by our model occur with rotations involving Ponatinib. Whereas a drug applied intermittently in a drug rotation is likely more well tolerated than if taken continuously, it seems unlikely that benefits would outweigh the risk for rotations involving Ponatinib. However, having shown that the potential could exist we recommend considering drug rotations if more well tolerated options are developed.

While no other malignancy fits this model quite as well as CML, there are similar resistance mutation phenomena in Gastrointestinal stromal tumour (GIST), $\mathrm{Ph}^{+}$acute lymphoblastic leukaemia (ALL), EGFR-mutant non small cell lung cancer (NSCLC), ALK-rearranged NSCLC, and other cancers. $\mathrm{Ph}^{+}$-ALL has the same molecular driver and develops resistance in a rather similar way [53]. In GIST, which is also treated with Imatinib, $50 \%-70 \%$ of late progression cases were suggested to be caused by mutations which affect drug binding interactions [54]. Both variants of NSCLC are also affected to some extent by kinase domain mutation induced resistance, though it accounts for a smaller fraction of observed cases $[55,56]$. For the last three, the spatial structure of solid tumours might also limit the applicability of this model, which does not include local competition effects, and/or differential exposure to drugs. We note though that these two are having the opposite influence, i.e., cells that out-compete others for development because they have better exposure to nutrients are also more accessible to the drugs, and hence the conclusions may be valid even for solid tumours.

\section{Additional file}

Additional file 1: Derivations of formulae, implementation details, justification of assumptions and figures too large for the main text. (PDF $277 \mathrm{~kb}$ )

\section{Abbreviations \\ ABL1: Abelson tyrosine kinase 1; ALK: Anaplastic lymphoma kinase; ALL: Acute lymphoblastic leukaemia; BCR: Breakpoint cluster region; CML: Chronic myeloid leukaemia; CSC: Cancer stem cell; EGFR: Epidermal growth factor receptor; GIST: Gastrointestinal stromal tumour; MMR: Major molecular response; NSCLC: Non small cell lung cancer; $\mathrm{Ph}^{+}$: Philadelphia chromosome positive; PSSM: Position specific scoring matrix; SNV: Single nucleotide variation; TKI: Tyrosine kinase inhibitor}

\section{Acknowledgements}

Not applicable

\section{Funding}

This work was supported by The Swedish Cancer Society (Cancerfonden) [CAN $2015 / 387$ to RF]. The funding body had no role in the design and execution of the study or in writing the manuscript.

\section{Availability of data and materials}

The simulation software, written in $\mathrm{C}++11$ and python 3 is platform independent and open source (zlib license) available at https://github.com/ Sandalmoth/wollsey-public. All usage in this article is compatible with release $\checkmark 1.00$. Specific dependencies and build instructions are included with the source code. 
The datasets used and/or analysed during the current study are available from the corresponding author on reasonable request.

\section{Authors' contributions}

The study was directed by RF. HJGL wrote software and performed research. ASdW did model design and analysis. HJGL wrote the manuscript with asstistance from RF and ASdW. All authors read and approved the final manuscript.

\section{Ethics approval and consent to participate \\ Not applicable}

\section{Consent for publication}

Not applicable

\section{Competing interests}

The authors declare that they have no competing interests.

\section{Publisher's Note}

Springer Nature remains neutral with regard to jurisdictional claims in published maps and institutional affiliations.

\section{Author details}

${ }^{1}$ Department of Chemistry and Biomedical Sciences, Linnæus University, 391 82 Kalmar, Sweden. ${ }^{2}$ Department of Mechanical and Industrial Engineering, Norwegian University of Science and Technology, 7491 Trondheim, Norway.

\section{Received: 27 August 2018 Accepted: 8 May 2019}

Published online: 28 May 2019

\section{References}

1. Ahronian LG, Corcoran RB. Strategies for monitoring and combating resistance to combination kinase inhibitors for cancer therapy. Genome Med. 2017;9(1):37. https://doi.org/10.1186/s13073-017-0431-3.

2. Fojo T. Commentary: Novel therapies for cancer: Why dirty might be better. The Oncologist. 2008;13(3):277-83. https://doi.org/10.1634/ theoncologist.2007-0090.

3. Friedman R. Drug resistance in cancer : Molecular evolution and compensatory proliferation. Oncotarget. 2016;7(11):11746-55. https:// doi.org/10.18632/oncotarget.7459.

4. Khorashad JS, Kelley TW, Szankasi P, Mason CC, Soverini S, Adrian LT, Eide CA, Zabriskie M, Lange T, Estrada J, Pomicter AD, Eiring A, Kraft IL, Anderson DJ, Gu Z, Alikian M, Reid AG, Foroni L, Marin D, Druker BJ, O'Hare T, Deininger M. BCR-ABL1 compound mutations in tyrosine kinase inhibitor-resistant CML: Frequency and clonal relationships. Blood. 2013;121(3):489-98. https://doi.org/10.1182/blood-2012-05-431379.

5. Buetti-Dinh A, Pivkin IV, Friedman R. S100A4 and its role in metastasis simulations of knockout and amplification of epithelial growth factor receptor and matrix metalloproteinases. Mol BioSyst. 2015;11:2247-54. https://doi.org/10.1039/C5MB00302D.

6. Friedman R, Boye K, Flatmark K. Molecular modelling and simulations in cancer research. BBA Rev Cancer. 2013;1836(1):1-14. https://doi.org/10. 1016/j.bbcan.2013.02.001.

7. Cook LM, Araujo A, Pow-Sang JM, Budzevich MM, Basanta D, Lynch CC. Predictive computational modeling to define effective treatment strategies for bone metastatic prostate cancer. Sci Rep. 2016;6:29384.

8. Deininger MWN, Goldman JM, Melo JV. The molecular biology of chronic myeloid leukemia. Blood. 2000;96(10):3343-56.

9. O'Hare T, Zabriskie M, Eiring A, W Deininger M. Pushing the limits of targeted therapy in chronic myeloid leukaemia. Nat Rev Cancer. 2012;12: 513-26.

10. O'Brien S, Guilhot F, Larson RA, Gathmann I, Baccarani M, Cervantes F, Cornelissen JJ, Fischer T, Hochhaus A, Hughes T, Lechner K, Nielsen JL, Rousselot P, Reiffers J, Saglio G, Shepherd J, Simonsson B, Gratwohl A, Goldman JM, Kantarjian H, Taylor K, Verhoef G, Bolton AE, Capdeville R, Druker BJ. Imatinib compared with interferon and low-dose cytarabine for newly diagnosed chronic-phase chronic myeloid leukemia. N Engl J Med. 2003;348(11):994-1004. https://doi.org/10.1056/NEJMoa022457.

11. Redaelli S, Mologni L, Rostagno R, Piazza R, Magistroni V, Ceccon M, Viltadi M, Flynn D, Gambacorti-Passerini C. Three novel patient-derived $B C R / A B L$ mutants show different sensitivity to second and third generation tyrosine kinase inhibitors. Am J Hematol. 2012;87(11):125-128. https://doi.org/10.1002/ajh.23338.

12. Hoy SM. Ponatinib: A review of its use in adults with chronic myeloid leukaemia or Philadelphia chromosome-positive acute lymphoblastic leukaemia. Drugs. 2014;74(7):793-806. https://doi.org/10.1007/s40265014-0216-6.

13. Lipton JH, Chuah C, Guerci-Bresler A, Rosti G, Simpson D, Assouline S, Etienne G, Nicolini FE, Le Coutre P, Clark R, Stenke L, Andorsky D, Oehler V, Lustgarten S, Rivera VM, Clackson T, Haluska FG, Baccarani M, Cortes J, Guilhot F, Hochhaus A, Hughes T, Kantarjian H, Shah N, Talpaz M, Deininger M. Epic: A phase 3 trial of ponatinib compared with imatinib in patients with newly diagnosed chronic myeloid leukemia in chronic phase (CP-CML). Blood. 2014;124(21):519-519.

14. Zabriskie M, Eide CA, Tantravahi SK, Vellore NA, Estrada J, Nicolini FE, Khoury HJ, Larson RA, Konopleva M, Cortes J, Kantarjian H, Jabbour E, Kornblau SM, Lipton JH, Rea D, Stenke L, Barbany G, Lange T, Hernández-Boluda J-C, Ossenkoppele GJ, Press RD, Chuah C, Goldberg SL, Wetzler M, Mahon F-X, Etienne G, Baccarani M, Soverini S, Rosti G, Rousselot $P$, Friedman $R$, Deininger $M$, Reynolds $K R$, Heaton $W L$, Eiring A, Pomicter AD, Khorashad JS, Kelley TW, Baron R, Druker BJ, Deininger M, O'Hare T. BCR-ABL1 compound mutations combining key kinase domain positions confer clinical resistance to ponatinib in $\mathrm{Ph}$ chromosome-positive leukemia. Cancer Cell. 2014;26(3):428-42. https:// doi.org/10.1016/j.ccr.2014.07.006.

15. O'Hare T, K Walters D, Stoffregen E, Jia T, W Manley P, Mestan J, Cowan-Jacob S, Lee F, Heinrich M, W N Deininger M, J Druker B. In vitro activity of bcr-abl inhibitors AMN107 and BMS-354825 against clinically relevant imatinib-resistant abl kinase domain mutants. Cancer Res. 2005;65:4500-5.

16. Weisberg E, W Manley P, Breitenstein W, Brüggen J, Cowan-Jacob S, Ray A, Huntly B, Fabbro D, Fendrich G, Hall-Meyers E, L Kung A, Mestan J, Q Daley G, Callahan L, Catley L, Cavazza C, Azam M, Mohammed A, Neuberg D, Griffin J. Characterization of AMN107, a selective inhibitor of native and mutant bcr-abl. Cancer Cell. 2005;7:129-41.

17. Cervantes F, Correa J-G, Pérez I., García-Gutiérrez V, Redondo S, Colomer D, Jiménez-Velasco A, Steegmann J-L, Sánchez-Guijo F, Ferrer-Marín F, Pereira A, Osorio S. Imatinib dose reduction in patients with chronic myeloid leukemia in sustained deep molecular response. Ann Hematol. 2017;96(1):81-5. https://doi.org/10.1007/s00277-016-2839-z.

18. Laneuville P. When to stop tyrosine kinase inhibitors for the treatment of chronic myeloid leukemia. Curr Treat Options Oncol. 2018;19:. https://doi. org/10.1007/s11864-018-0532-2.

19. Bozic I, Reiter JG, Allen B, Antal T, Chatterjee K, Shah P, Moon YS, Yaqubie A, Kelly N, Le DT, Lipson EJ, Chapman PB, Diaz J, Luis A, Vogelstein B, Nowak MA. Evolutionary dynamics of cancer in response to targeted combination therapy. eLife. 2013;2:00747. https://doi.org/10. 7554/eLife.00747.

20. Kimmel M, Axelrod DE. Branching Processes in Biology. Ecological Studies. New York: Springer; 2002. https://books.google.se/books?id= B2udCdiB-olC.

21. Danesh K, Durrett R, Havrilesky LJ, Myers E. A branching process model of ovarian cancer. J Theor Biol. 2012;314(Supplement C):10-5. https://doi. org/10.1016/j.jtbi.2012.08.025.

22. Altrock PM, Liu L, Michor F. The mathematics of cancer: Integrating quantitative models. Nat Rev Cancer. 2015;15:730-45.

23. Li C, Wang J. Quantifying the landscape for development and cancer from a core cancer stem cell circuit. Cancer Res. 2015;75(13):2607-18. https://doi.org/10.1158/0008-5472.CAN-15-0079.

24. Michor F, Hughes TP, Iwasa Y, Branford S, Shah NP, Sawyers CL, Nowak MA. Dynamics of chronic myeloid leukaemia. Nature. 2005;435:1267-70. https://doi.org/10.1038/nature03669.

25. Dingli D, M Pacheco J, Traulsen A. Multiple mutant clones in blood rarely coexist. Phys Rev E Stat Nonlinear Soft Matter Phys. 2008;77:021915. https://doi.org/10.1103/PhysRevE.77.021915.

26. Abraham S, Hopcroft L, Carrick E, Drotar M, Dunn K, Williamson A, Korfi K, Baquero P, E. Park L, Scott M, Pellicano F, Pierce A, Copland M, Nourse C, Grimmond S, Vetrie D, Whetton A, Holyoake T. Dual targeting of p53 and c-MYC selectively eliminates leukaemic stem cells. Nature. 2016;534:341-6.

27. Dingli D, Traulsen A, Pacheco JM. Chronic myeloid leukemia: Origin, development, response to therapy, and relapse. Clin Leuk. 2008;2(2): 133-9. https://doi.org/10.3816/CLK.2008.n.017. 
28. Jørgensen $\mathrm{HG}$, Allan E, Jordanides NE, Mountford JC, Holyoake TL. Nilotinib exerts equipotent antiproliferative effects to imatinib and does not induce apoptosis in CD34+ CML cells. Blood. 2007;109(9):4016-9. https://doi.org/10.1182/blood-2006-11-057521.

29. Graham SM, Jørgensen HG, Allan E, Pearson C, Alcorn MJ, Richmond L, Holyoake TL. Primitive, quiescent, Philadelphia-positive stem cells from patients with chronic myeloid leukemia are insensitive to STI571 in vitro. Blood. 2002;99(1):319-25. https://doi.org/10.1182/blood.V99.1.319.

30. Leder K, Foo J, Skaggs B, Gorre M, Sawyers CL, Michor F. Fitness conferred by $B C R-A B L$ kinase domain mutations determines the risk of pre-existing resistance in chronic myeloid leukemia. PLOS ONE. 2011;6(11):1-11. https://doi.org/10.1371/journal.pone.0027682.

31. Dingli D, Traulsen A, M Pacheco J. Report stochastic dynamics of hematopoietic tumor stem cells. Cell Cycle (Georgetown, Tex). 2007;6: 461-6. https://doi.org/10.4161/cc.6.4.3853.

32. Kantarjian H, Pasquini R, Hamerschlak N, Rousselot P, Holowiecki J, Jootar S, Robak T, Khoroshko N, Masszi T, Skotnicki A, Hellmann A, Zaritsky A, Golenkov A, Radich J, Hughes T, Countouriotis A, Shah N. Dasatinib or high-dose imatinib for chronic-phase chronic myeloid leukemia after failure of first-line imatinib: A randomized phase 2 trial. Blood. 2007;109(12):5143-50. https://doi.org/10.1182/blood-2006-11056028.

33. Shah $\mathrm{N}$, Kantarjian $\mathrm{H}$, Kim D-W, Réa D, Dorlhiac-Llacer PE, Milone JH, Vela-Ojeda J, Silver RT, Khoury HJ, Charbonnier A, Khoroshko N, Paquette RL, Deininger M, Collins RH, Otero I, Hughes T, Bleickardt E, Strauss L, Francis S, Hochhaus A. Intermittent target inhibition with dasatinib $100 \mathrm{mg}$ once daily preserves efficacy and improves tolerability in imatinib-resistant and -intolerant chronic-phase chronic myeloid leukemia. J Clin Oncol. 2008;26(19):3204-12. https://doi.org/10.1200/JCO. 2007.14.9260

34. Soverini S, Benedittis C, Papayannidis C, Machova Polakova K, Venturi C, Russo D, Bresciani P, lurlo A, Mancini M, Vitale A, Chiaretti S, Foà R, Abruzzese E, Sora F, Kohlmann A, Haferlach T, Baccarani M, Cavo M, Giovanni M. Clinical impact of low burden BCR-ABL1 mutations detectable by amplicon deep sequencing in Philadelphia-positive acute lymphoblastic leukemia patients. Leukemia. 2016;30: https://doi.org/10. 1038/leu.2016.17

35. Marusyk A, Almendro V, Polyak K. Intra-tumour heterogeneity: A looking glass for cancer? Nat Rev Cancer. 2012;12:323-34.

36. O'Hare T, Eide CA, Deininger MWN. BCr-abl kinase domain mutations, drug resistance, and the road to a cure for chronic myeloid leukemia. Blood. 2007;1 10(7):2242-9. https://doi.org/10.1182/blood-2007-03066936.

37. Gambacorti-Passerini C, Rossi F, Verga M, Ruchatz H, Gunby R, Frapolli R, Zucchetti M, Scapozza L, Bungaro S, Tornaghi L, Rossi F, Pioltelli P, Pogliani E, D'Incalci M, Corneo G. Differences between in vivo and in vitro sensitivity to imatinib of $\mathrm{Bcr} / \mathrm{Abl}+$ cells obtained from leukemic patients. Blood Cells Mol Dis. 2002;28(3):361-72. https://doi.org/10.1006/ bcmd.2002.0526.

38. Gambacorti-Passerini CB, Gunby RH, Piazza R, Galietta A, Rostagno R, Scapozza L. Molecular mechanisms of resistance to imatinib in Philadelphia-chromosome-positive leukaemias. Lancet Oncol. 2003;4(2): 75-85. https://doi.org/10.1016/S1470-2045(03)00979-3.

39. Liu C, Liu Z, Wang J. Uncovering the molecular and physiological processes of anticancer leads binding human serum albumin: A physical insight into drug efficacy. PLoS ONE. 2017;12(4):1-22. https://doi.org/10. 1371/journal.pone.0176208.

40. Demetri G, Lo Russo P, Macpherson I, Wang D, A Morgan J, Brunton V, Phd P, Agrawal S, Voi M, Evans J. Phase i dose-escalation and pharmacokinetic study of dasatinib in patients with advanced solid tumors. Clin Cancer Res. 2009;15:6232-40. https://doi.org/10.1158/10780432.CCR-09-0224.

41. Pharmaceutical Specialists in Sweden. http://fass.se. Accessed 20 Jan 2019.

42. Moran PAP. Random processes in genetics. Math Proc Cambridge Philos Soc. 1958;54(1):60-71. https://doi.org/10.1017/S0305004100033193.

43. White D, Saunders V, Grigg A, Arthur C, Filshie R, Leahy MF, Lynch K, To $L B$, Hughes T. Measurement of in vivo BCR-ABL kinase inhibition to monitor imatinib-induced target blockade and predict response in chronic myeloid leukemia. J Clin Oncol. 2007;25(28):4445-51. https://doi. org/10.1200/JCO.2006.09.9499.
44. Hanfstein B, Müller M, Hehlmann R, Erben P, Lauseker M, Fabarius A, Schnittger S, Haferlach C, Göhring G, Proetel U, Kolb H-J, W Krause S, Hofmann W-K, Schubert J, Einsele H, Dengler J, Hänel M, Falge C, Kanz $L$, Hochhaus A. Early molecular and cytogenetic response is predictive for long-term progression-free and overall survival in chronic myeloid leukemia (CML). Leukemia. 2012;26:2096-102.

45. Dingli D, Traulsen A, Pacheco JM. Compartmental architecture and dynamics of hematopoiesis. PLoS ONE. 2007;2(4):1-4. https://doi.org/10. 1371/journal.pone.0000345.

46. Valent $P$, Herndlhofer S, Schneeweiß M, Boidol B, Ringler A, Kubicek S, V. Gleixner K, Hoermann G, Hadzijusufovic E, Müllauer L, R. Sperr W, Superti-Furga G, Mannhalter C. TKI rotation-induced persistent deep molecular response in multi-resistant blast crisis of ph+ CML. Oncotarget. 2017:8:23061-72.

47. Gugliotta G, Castagnetti F, Breccia M, Gozzini A, Usala E, Carella A, Rege-Cambrin G, Martino B, Abruzzese E, Albano F, Stagno F, Luciano L, D'Adda M, Bocchia M, Cavazzini F, Tiribelli M, Lunghi M, Pia Falcone A, Musolino C, Baccarani M. Rotation of nilotinib and imatinib for first-line treatment of chronic phase chronic myeloid leukemia. Am J Hematol. 2016;91:617-22.

48. Cvijović l, Good BH, Jerison ER, Desai MM. Fate of a mutation in a fluctuating environment. Proc Natl Acad Sci U S A. 2015;112(36):5021-8. https://doi.org/10.1073/pnas.1505406112.

49. Soverini S, Rosti G, lacobucci I, Baccarani M, Martinelli G. Choosing the best second-line tyrosine kinase inhibitor in imatinib-resistant chronic myeloid leukemia patients harboring bcr-abl kinase domain mutations: How reliable is the IC50?. The Oncologist. 2011;16(6):868-76. https://doi. org/10.1634/theoncologist.2010-0388.

50. Jabbour E, Jones D, Kantarjian H, O'Brien S, Tam C, Koller C, Burger JA, Borthakur G, Wierda WG, Cortes J. Long-term outcome of patients with chronic myeloid leukemia treated with second-generation tyrosine kinase inhibitors after imatinib failure is predicted by the in vitro sensitivity of BCR-ABL kinase domain mutations. Blood. 2009;1 14(10): 2037-43. https://doi.org/10.1182/blood-2009-01-197715.

51. Chakrabarti $S$, Michor F. Pharmacokinetics and drug interactions determine optimum combination strategies in computational models of cancer evolution. Cancer Res. 2017;77(14):3908-21. https://doi.org/10. 1158/0008-5472.CAN-16-2871.

52. Foo J, Michor F. Evolution of resistance to anti-cancer therapy during general dosing schedules. J Theor Biol. 2010;263(2):179-88. https://doi. org/10.1016/j.jtbi.2009.11.022.

53. Pfeifer H, Wassmann B, Pavlova A, Wunderle L, Oldenburg J, Binckebanck A, Lange T, Hochhaus A, Wystub S, Brück P, Hoelzer D, Ottmann OG. Kinase domain mutations of BCR-ABL frequently precede imatinib-based therapy and give rise to relapse in patients with de novo Philadelphia-positive acute lymphoblastic leukemia (Ph+ ALL). Blood. 2007;110(2):727-34. https://doi.org/10.1182/blood-2006-11-052373.

54. Sleijfer S, Wiemer E, Seynaeve C, Verweij J. Improved insight into resistance mechanisms to imatinib in gastrointestinal stromal tumors: A basis for novel approaches and individualization of treatment. The Oncologist. 2007;12(6):719-26. https://doi.org/10.1634/theoncologist.126-719.

55. Doebele RC, Pilling AB, Aisner DL, Kutateladze TG, Le AT, Weickhardt AJ, Kondo KL, Linderman DJ, Heasley LE, Franklin WA, Varella-Garcia M, Camidge DR. Mechanisms of resistance to crizotinib in patients with ALK gene rearranged non-small cell lung cancer. Clin Cancer Res. 2012;18(5): 1472-82. https://doi.org/10.1158/1078-0432.CCR-11-2906.

56. Lin $Y$, Wang $X$, Jin H. EGFR-TKI resistance in NSCLC patients: Mechanisms and strategies. Am J Cancer Res. 2014;4(5):411-35. 